\title{
COVID-19: Screening and triage challenges in people with disability due to Spinal Cord Injury
}

\author{
Radha Korupolu ${ }^{1,2} \cdot$ Argyrios Stampas $^{1,2} \cdot$ Carlee Gibbons $^{3}$ - Isaac Hernandez Jimenez ${ }^{1,2}$ - Felicia Skelton ${ }^{4,5}$. \\ Monica Verduzco-Gutierrez $\mathbb{1}^{3}$
}

Received: 2 April 2020 / Revised: 20 April 2020 / Accepted: 21 April 2020

(C) International Spinal Cord Society 2020

\begin{abstract}
An outbreak of a novel coronavirus disease (COVID-19) that emerged in the Chinese city of Wuhan has rapidly become a global public health pandemic. As of March 2020, the Centers for Disease Control and Prevention (CDC) has described priority levels for testing patients with suspected COVID-19 and information on when to seek medical attention. However, there is a paucity of further guidance for people with spinal cord injury (SCI) who may not present with typical symptoms of COVID-19 due to altered physiology. This may pose challenges with surveillance, risk stratification, and initial management of this vulnerable population. In this point-counterpoint discussion, we outline important differences in presentation along with COVID-19 cases co-morbid with SCI.
\end{abstract}

People with spinal cord injury (SCI) represent a unique diagnostic challenge as it relates to coronavirus disease COVID-19 pandemic. Individuals with SCI develop a myriad of physiological changes that not only increase their risk of morbidity from COVID-19, but may also mask the presentation of an acute respiratory illness which can potentially delay the diagnosis of COVID-19. These include temperature dysregulation (poikilothermia), impaired cough, and abnormal sensations at or below the

These authors contributed equally: Radha Korupolu, Argyrios Stampas

Radha Korupolu

radha.korupolu@uth.tmc.edu

1 Department of Physical Medicine and Rehabilitation, McGovern Medical School, The University of Texas Health Science Center, Houston, TX, USA

2 The Institute of Rehabilitation \& Research (TIRR) Memorial Hermann, Houston, TX, USA

3 Department of Rehabilitation Medicine, Long School of Medicine, The University of Texas Health Science Center, San Antonio, TX, USA

4 Center for Innovations in Quality, Effectiveness and Safety, Michael E. DeBakey VA Medical Center, Houston, TX, USA

5 H. Ben Taub Department of Physical Medicine and Rehabilitation, Baylor College of Medicine, Houston, TX, USA neurological level of injury (NLI) [1-4]. In those with NLI at or above thoracic level 6 (T6), noxious simulation including infection below the NLI may result in autonomic dysreflexia (AD), a medical emergency with symptoms including: elevated blood pressure, low heart rate, chest tightness, facial flushing, profuse sweating, vision changes, and pounding headache [5]. In addition, individuals with SCI may develop nonspecific symptoms during infection, including new or worsening spasticity, neuropathic pain, $\mathrm{AD}$ and/or fatigue, not consistent with the current Centers for Disease Control and Prevention (CDC) guidelines for COVID-19 [6-8]. In an international survey of health care professionals $(n=783)$ who care for individuals with SCI, $10.3 \%$ reported their patients with COVID-19 had increased spasticity, $6.9 \%$ reported that their patients had rigors, and $6.9 \%$ reported that their patients had been asymptomatic. This early report warrants special consideration during triage of people with SCI for suspected COVID-19 [9].

As the COVID-19 pandemic continues to evolve, local health departments have been tasked with providing updates by region. The CDC, as of March 29th, 2020, delineated priority level groups for COVID-19 testing [10] and acknowledged extra considerations required for individuals with disabilities [11]. However, specific risk stratification and/ or testing guidelines for people with SCI are not included. As of March 29th, 2020, the CDC lists the following symptoms of COVID-19: fever, cough, and shortness of breath, with emergency symptoms of trouble breathing, persistent pain or 
pressure in the chest, new confusion or inability to arouse, bluish lips or face [12]. The following describes how the presentation of these symptoms may be altered in people with SCI.

People with SCI may lack the febrile response because of the interruption of the connection between the hypothalamus and efferent nerves (sympathetic and motor) responsible for raising core temperature and may delay diagnostic workup [4, 5]. Temperature dysregulation has shown to be proportional to the NLI, with persons with tetraplegia being more impaired than persons with paraplegia [4]. Though urinary tract infections (UTIs) are the most common cause of fever and emergency room visits in persons with SCI who have an indwelling catheter, a positive urine analysis (UA) should not stop further investigation of a fever in the setting of associated respiratory symptoms [3] given the high likelihood this represents asymptomatic bacteriuria without any other focal signs of infection [13].

SCI patients with COVID-19 may not present with cough due to disruption of normal respiratory physiology. The ability to produce an effective cough is severely impaired in patients with cervical or high thoracic SCI due to loss of innervation to the abdominal and/or intercostal muscles [2, 14, 15]. Individuals with SCI exhibit reduced lung volumes and flow rates as a result of respiratory muscle weakness including diaphragm and intercostal muscles [7, 8]. Changes in spirometric measurements in SCI are dependent on NLI and posture. As the NLI ascends, total lung capacity is progressively reduced resulting in atelectasis which can confound findings of pneumonia on imaging. Given an already reduced lung capacity and impaired cough, a high level of suspicion for respiratory illness is needed to ensure an early diagnosis, and close monitoring is needed even in a mild-to-moderate clinical presentation of a positive COVID-19 case. Accessory muscle use is a sign of respiratory muscle fatigue and impending respiratory failure in people with SCI. Other common factors that can exacerbate or cause SOB include severe spasticity, severe constipation, and anxiety. Importantly, some persons with SCI are ventilator dependent at baseline. These home units are capable of adjustments in the setting of respiratory illness. Given the concerns of worldwide ventilator shortages, we recommend that people with SCI continue to use their personal ventilators during COVID-19 treatment.

Patients with SCI may not perceive symptoms the CDC would classify as an even more alarming presentation of COVID-19, including chest pain or pressure. Individuals with cervical or high thoracic SCI may not have chest sensations, often absent even in the extreme case of myocardial infarction [16, 17]. This may extend to myocarditis seen in COVID-19 [18]. Although the chest pain may not be sensed, it can result in AD which can in turn worsen cardiac symptoms [19]. Providers must be mindful of this clinical conundrum. AD requires further mention, as it can lead to non-respiratory illness related $\mathrm{CP}$ and/or SOB. In serious cases, the uncontrolled hypertension can result in loss of consciousness, cerebral and spinal subarachnoid hemorrhage, seizures, and pulmonary edema. It is a potentially life-threatening condition [20-22]. A variety of noxious stimuli below the level of injury can trigger an episode. It is most commonly caused by irritation of the bladder or bowel. The detailed algorithm for management of $\mathrm{AD}$ is described elsewhere [21]. Immediate removal of noxious stimuli and monitoring blood pressures with use of fastacting, as needed short-duration antihypertensive medications, are important to prevent morbidity from AD.

The differential for new confusion or inability to arouse in a patient with a SCI must remain broad. SCI specific common causes of altered mental status (AMS) include UTI, pneumonia, orthostatic hypotension, and AD [20]. AD-related complications can also cause AMS as stated above. These must be part of the differential diagnosis as the person under investigation for COVID-19 is assessed. In addition, there are SCI-specific concerns for etiologies of blueish lips and face, the final symptom or sign the CDC lists as a possible and emergent COVID-19 clinical picture. Specific to the SCI population, one must consider increased demand for oxygen despite the inability for forceful gasps of air or abdominal accessory muscle recruitment. Furthermore, these individuals are at risk for cardiac compromise from AD. In addition, poikilothermia can lead to this finding too. None of the above excludes the possibility of an individual experiencing a significant respiratory illness.

As the numbers of COVID-19 cases climb worldwide, scenarios describing the above diagnostic challenges are arising in the literature. In the first recently published case report from Italy, a male patient with chronic C4 tetraplegia was diagnosed with COVID-19. His diagnosis was delayed due to UA suggestive of UTI, atypical presentation with no cough, and chest X-ray findings that were obscured with elevated hemidiaphragm [23]. In our own experiences, we had a 40-year-old male with traumatic SCI (C5 motor level) and brain injury who developed increased respiratory demands and a requirement for supplemental oxygen shortly after admission to our inpatient rehabilitation facility (IRF). He had fevers (38.6-39.2 ${ }^{\circ} \mathrm{C}$ ), tachypnea, chest tightness, shortness of breath, and excessive secretions. UA was negative for UTI, laboratory workup showed lymphopenia and chest X-ray revealed possible pneumonia versus atelectasis. The above findings raised concerns for COVID19. He was transferred back to acute care where COVID-19 test was negative. On review of records from the acute care hospital prior to admission to our IRF, he had elevated temperature $\left(37.7^{\circ} \mathrm{C}\right)$ in spite of being on scheduled antipyretics. His fever workup for UTI, bacteremia, and pneumonia at the acute care hospital was negative for any infectious etiology at that time. In this case the important 
take home point is that acute cervical SCI patients can present with signs and symptoms that mimic COVID-19. A COVID-19 test in acute care hospital prior to transfer to IRF where there are likely inadequate resources to manage such cases could have prevented unnecessary urgent transfer back to acute care hospital. In another case of a 78-year-old male with incomplete paraplegia (T4 neurological level) from spinal cord stimulator complication was admitted to IRF where he developed fever on Day 4. Fevers were attributed to a UTI after workup and treated with antibiotics. $\mathrm{He}$ also developed loose stools which were attributed to bowel regimen for neurogenic bowel and antibiotic usage. A few days later fevers relapsed with intermittent cough. Due to persistent fever and cough computed tomography scan of chest was done which showed ground-glass opacities in the lungs. He was immediately transferred back to acute care hospital where he was tested positive for COVID-19. His diagnosis was delayed by 2 weeks from initial onset of fevers.

The SCI population poses a unique diagnostic challenge in the current pandemic environment. Given the altered physiology described in SCI, atypical presentation of a respiratory illness and COVID-19 requires a heightened suspicion index for COVID-19 infection. The prompt involvement of an SCI-trained physician, whether in-person or virtual, when assessing a patient with new or worsening symptomatology is warranted and advised during the COVID-19 pandemic. The COVID-19 pandemic has forced rapid and drastic changes in health care delivery; many clinicians are turning to telemedicine as a potential stopgap to provide urgent outpatient management to their patients, including screening for possible COVID-19 symptoms [24]. Unfortunately, the same health disparities that people with SCI and other disabilities face at baseline may hinder their access to this technology as well [25]. In conclusion, we believe the most appropriate global guidance for persons with SCI is to include atypical symptomatology for COVID-19 symptoms and expand the criteria for testing.

Regarding future emergency situations or pandemics, we will be able to adapt rapidly if we optimize the many lessons learned from COVID-19. Contingency plans should be readily available to help facilitate improved outcomes for our patients with disabilities.

\section{Compliance with ethical standards}

Conflict of interest The authors declare that they have no conflict of interest.

Publisher's note Springer Nature remains neutral with regard to jurisdictional claims in published maps and institutional affiliations.

\section{References}

1. Berlowitz DJ, Wadsworth B, Ross J. Respiratory problems and management in people with spinal cord injury. Breathe. 2016;12:328-40. https://doi.org/10.1183/20734735.012616.

2. Cardozo CP. Respiratory complications of spinal cord injury. $\mathbf{J}$ Spinal Cord Med. 2007;30:307-8. https://doi.org/10.1080/ 10790268.2007.11753945.

3. Price MJ, Trbovich M. Thermoregulation following spinal cord injury. Handb Clin Neurol. 2018;157:799-820. https://doi.org/10. 1016/B978-0-444-64074-1.00050-1.

4. Sezer N, Akkus S, Ugurlu FG. Chronic complications of spinal cord injury. World J Orthop. 2015;6:24-33. https://doi.org/10. 5312/wjo.v6.i1.24.

5. Biering-Sorensen F, Biering-Sorensen $\mathrm{T}$, Liu N, Malmqvist L, Wecht JM, Krassioukov A. Alterations in cardiac autonomic control in spinal cord injury. Auton Neurosci 2018;209:4-18. https://doi.org/10.1016/j.autneu.2017.02.004.

6. Cardenas DD, Hooton TM. Urinary tract infection in persons with spinal cord injury. Arch Phys Med Rehabil. 1995;76:272-80. https://doi.org/10.1016/s0003-9993(95)80615-6.

7. Garcia Leoni ME, Esclarin De, Ruz A. Management of urinary tract infection in patients with spinal cord injuries. Clin Microbiol Infect. 2003;9:780-5. https://doi.org/10.1046/j.1469-0691.2003.00643.x.

8. Montgomerie JZ. Infections in patients with spinal cord injuries. Clin Infect Dis. 1997;25:1285-90. https://doi.org/10.1086/516144.

9. Stillman MD, Capron M, Alexander M, Di Giusto ML, Scivoletto G. COVID-19 and spinal cord injury and disease: results of an international survey. Spinal Cord Ser Cases. 2020;6:21. https:// doi.org/10.1038/s41394-020-0275-8.

10. Priorities for testing patients with suspected covid-19 infection. 2020. https://www.cdc.gov/coronavirus/2019-ncov/hcp/clinical-criteria.html.

11. World Health Organization. Disability considerations during the COVID-19 outbreak. 2020. https://www.who.int/internal-publica tions-detail/disability-considerations-during-the-covid-19-outbreak.

12. Centers for Disease Control and Prevention. Evaluating and Testing Persons for Coronavirus Disease 2019 (COVID-19). https://www.cdc.gov/coronavirus/2019-ncov/hcp/clinical-criteria. html.

13. Nicolle LE, Gupta K, Bradley SF, et al. Clinical Practice Guideline for the Management of Asymptomatic Bacteriuria: 2019 update by the Infectious Diseases Society of America. Clin Infect Dis. 2019;68:1611-5. https://doi.org/10.1093/cid/ciz021.

14. Lemons VR, Wagner FC Jr. Respiratory complications after cervical spinal cord injury. Spine. 1994;19:2315-20. https://doi.org/ 10.1097/00007632-199410150-00011.

15. Tollefsen E, Fondenes O. Respiratory complications associated with spinal cord injury. Tidsskr Nor Laegeforen. 2012;132:1111-4. https://doi.org/10.4045/tidsskr.10.0922.

16. Walker WC, Khokhar MS. Silent cardiac ischemia in cervical spinal cord injury: case study. Arch Phys Med Rehabil. 1992;73:91-4.

17. Yu AJ, Lee MY, Blankenship LD. Non-ST segment elevation myocardial infarction presenting with dyspnea on exertion in the absence of chest pain in a spinal cord injury patient: a case report. Arch Phys Med Rehabil. 2005;86:E42. https://doi.org/10.1016/j. apmr.2005.07.240.

18. Rodriguez-Morales AJ, Cardona-Ospina JA, Gutierrez-Ocampo E, et al. Clinical, laboratory and imaging features of COVID-19: a systematic review and meta-analysis. Travel Med Infect Dis. 2020:101623. https://doi.org/10.1016/j.tmaid.2020.101623.

19. Htwe OCT, Yin KN, Hussain RI, Naicker AS. Unusual presentation of autonomic dysreflexia in patient with cervical spinal cord injury-is myocardial infarction a cause or an effect? In 4th 
International Congress on Neurology and Epidemiology. Kuala Lumpur, Malaysia: Neuroepidemiology; 2014. p. 71-113.

20. Furlan JC. Autonomic dysreflexia: a clinical emergency. J Trauma Acute Care Surg. 2013;75:496-500. https://doi.org/10.1097/TA. 0b013e31829fda0a.

21. Consortium for Spinal Cord Medicine. Acute management of autonomic dysreflexia: individuals with spinal cord injury presenting to health-care facilities. J Spinal Cord Med. 2002;25: Suppl 1:S67-88.

22. Krassioukov A, Warburton DE, Teasell R, Eng JJ. Spinal Cord Injury Rehabilitation Evidence Research Team. A systematic review of the management of autonomic dysreflexia after spinal cord injury. Arch Phys Med Rehabil. 2009;90:682-95. https://doi. org/10.1016/j.apmr.2008.10.017.

23. Righi G, Del Popolo G. COVID-19 tsunami: the first case of a spinal cord injury patient in Italy. Spinal Cord Ser Cases.2020;6:22 https://doi.org/10.1038/s41394-020-0274-9.

24. Mehrotra, A Ray, K, Brockmeyer, DM, et al. Rapidly converting to "Virtual Practices": outpatient care in the era of Covid-19. NEJM Catal. 2020. www.catalyst.nejm.org. https://doi.org/10. 1056/CAT.20.0091.

25. Armitage R, Nellums LB. The COVID-19 response must be disability inclusive. Lancet. Public Health 2020;S2468-2667: 30076-1. 